\title{
Cerebral perfusion imaging in vasospasm
}

\author{
Shivanand P. Lad, M.D., Ph.D., Raphael Guzman, M.D., Michael E. Kelly, M.D., \\ GoRdon Li, M.D., Michael LiM, M.D., KaRL LovbaLD, M.D., \\ and Gary K. Steinberg, M.D., Ph.D.
}

Department of Neurosurgery, Stanford University School of Medicine, Stanford, California

\begin{abstract}
$\checkmark$ Vasospasm following cerebral aneurysm rupture is one of the most devastating sequelae and the most common cause of delayed ischemic neurological deficit (DIND). Because vasospasm also is the most common cause of morbidity and mortality in patients who survive the initial bleeding episode, it is imperative not only to diagnose the condition but also to predict which patients are likely to become symptomatic. The exact pathophysiology of vasospasm is complex and incompletely elucidated. Early recognition of vasospasm is essential because the timely use of several therapeutic interventions can counteract this disease and prevent the occurrence of DIND. However, the prompt implementation of these therapies depends on the ability to predict impending vasospasm or to diagnose it at its early stages.

A number of techniques have been developed during the past several decades to evaluate cerebral perfusion, including positron emission tomography, xenon-enhanced computed tomography, single-photon emission computed tomography, perfusion- and diffusion-weighted magnetic resonance imaging, and perfusion computed tomography. In this article, the authors provide a general overview of the currently available perfusion imaging techniques and their applications in treating vasospasm after a patient has suffered a subarachnoid hemorrhage. The use of cerebral perfusion imaging techniques for the early detection of vasospasm is becoming more common and may provide opportunities for early therapeutic intervention to counteract vasospasm in its earliest stages and prevent the occurrence of DINDs.
\end{abstract}

\section{KEY WORDS • vasospasm • subarachnoid hemorrhage • perfusion imaging • cerebral blood flow • transcranial Doppler ultrasonography • neurosurgery}

$\mathrm{V}$ ASOSPASM following cerebral aneurysm rupture is one of the most devastating sequelae and is also the most common cause of DIND. Vasospasm can be demonstrated using angiography in approximately 60 to $70 \%$ of patients with ruptured aneurysms. ${ }^{29,31,38}$ Clinical vasospasm resulting in DIND occurs in 20 to $30 \%$ of patients within 3 to 14 days after aneurysmal SAH. Given that vasospasm is the most common cause of morbidity and mortality in patients who survive the initial bleeding episode, it is imperative not only to diagnose the condition but also to predict which patients are likely to become symptomatic. The pathophysiological mechanisms of vasospasm are complex and incompletely elucidated. The presence of oxyhemoglobin in the subarachnoid cisterns seems to be a key promoter of the phenomena that ultimately cause narrowing of the arterial lumen and impaired autoregulation. $24,26,31,32,36,38,39$ The vascular changes are typically reversible: when severe, however, they may cause cerebral infarction and DIND. The amount of blood in the subarachnoid space on the initial brain CT scan, age youn-

Abbreviations used in this paper: $\mathrm{ACA}=$ anterior cerebral artery; $\mathrm{CBF}=$ cerebral blood flow; $\mathrm{CBV}=$ cerebral blood volume; $\mathrm{CT}=$ computed tomography; DIND = delayed ischemic neurological deficit MCA = middle cerebral artery $; \mathrm{MR}=$ magnetic resonance; $\mathrm{PET}=$ positron emission tomography; $\mathrm{SAH}=$ subarachnoid hemorrhage; SPECT = single-photon emission computed tomography; $\mathrm{TCD}=$ transcranial Doppler. ger than 70 years, and a history of smoking are risk factors for vasospasm, but their predictive value is limited. . $^{31,38}$

The most widely used monitoring technique for the detection of vasospasm is TCD ultrasonography, which was introduced in the early 1980s by Aaslid and colleagues. ${ }^{1,2}$ A demonstration of elevated blood flow velocity and an increase in turbulent flow on TCD ultrasonography can provide an early clinical awareness of vasospasm that involves the circle of Willis. ${ }^{5,41}$ Because this method is noninvasive and can be repeated as often as necessary, it is commonly used to monitor for cerebrovascular vasospasm after SAH. However, TCD ultrasonography can fail to detect vasoconstriction if vasospasm occurs beyond insonated arteries, such as in the distal $\mathrm{M}_{2}$ (MCA), the $\mathrm{A}_{2}$ (ACA), the $\mathrm{P}_{2}$ (posterior cerebral artery), or the vertebrobasilar system. In cases of severe vasospasm, decreases in CBF can occur that lead to a drop in measurable blood velocity. Also, increased intracranial pressure or brain edema can increase the pulsatility of the waveform, which makes interpretation of higher velocities difficult. Patient movement, suboptimal insonation windows, aberrant vessel course, and clip artifacts can further inhibit the detection of pathological signals.

The diagnosis of vasospasm after SAH is often made using a combination of patient history, physical examination, CT scanning to determine the extent of rupture, and TCD ultrasonography, along with other imaging modalities such as CT angiography, MR imaging, MR angiography, and catheter angiography. Despite multimodality an- 
alysis of vasospasm, the ability to predict DIND is still limited. In a recent study, investigators demonstrated that when both TCD ultrasonography and catheter angiography revealed vasospasm, the positive predictive value for the occurrence of cerebral infarction was $67 \% .{ }^{32}$ Conversely, when both studies indicated an absence of vasospasm, the negative predictive value of this combination was $72 \%$. Early recognition of vasospasm and the potential risk of DIND is essential because the rapid implementation of standard therapies such as triple-H (hypertension, hypervolemia, and hemodilution) therapy, intraarterial vasodilator application, and balloon angioplasty serves to counteract vasospasm and prevent the occurrence of DIND. ${ }^{24,26,29,38}$ However, the prompt use of these therapies depends on the ability to predict impending vasospasm or to diagnose it at its early stages.

A number of techniques to evaluate cerebral perfusion have been developed during the past several decades, beginning in the 1970s with PET and Xe-CT scanning. During the next two decades, other perfusion imaging techniques were introduced, including SPECT scanning, perfusion- and diffusion-weighted MR imaging, and perfusion CT scanning (Table 1). These techniques have been used to evaluate a variety of disease states, including acute and chronic ischemia and ischemia from post-SAH vasospasm. In this article we provide a general overview of the currently available perfusion imaging techniques and their application in patients with post-SAH vasospasm.

\section{Cerebral Perfusion Imaging}

\section{The Xe-CT Modality}

Xenon is a biologically inert gas that is soluble in both lipid and water and is freely diffusible. These attributes make it useful in the evaluation of cerebral perfusion because in its gaseous state, it dissolves in blood after inhalation and freely crosses the blood-brain barrier into the brain parenchyma. Measurement of the tissue concentration of xenon then reflects both arterial input to and uptake by the target tissue. Its radioactive form, xenon133 , has been used for cerebral perfusion evaluation for more than 30 years. ${ }^{22}$ Because $\mathrm{x}$-ray attenuation by xenon is similar to that by iodine, it can be used as a contrast agent for sensitive X-ray based technology such as CT, in which case the neuroimaging procedure is known as XeCT scanning. Therefore, the nonradioactive form has been adopted for use as an inhaled, diffusible tracing agent during $\mathrm{CT}$ scanning to measure tissue perfusion in patients. ${ }^{20,43,45}$

The technique requires the inhalation of a mixture of stable xenon gas and oxygen. With improvements in CT scanner technology, the amount of xenon required for an adequate contrast-to-noise ratio has decreased. Currently, a mixture of $28 \%$ xenon and $72 \%$ oxygen is most commonly used, with inhalation for a period of 4.3 minutes. ${ }^{42}$ Following the acquisition of a nonenhanced baseline scan of the entire head, six contiguous 10-mm-thick levels are selected for study, with each level scanned six times during the inhalation period, which requires exact table relocation. As the xenon accumulates in cerebral tissues, the patient experiences a sedative effect. After inhalation ceases, a few minutes are required for the gas to be washed out of the cerebral tissues and the patient to return to baseline attentiveness. Processing of the data allows the generation of CBF maps, from which quantitative information can be extracted..$^{30}$

Investigators in multiple studies have validated the accuracy of CBF values obtained using Xe-CT scanning., ${ }^{9,20}$, ${ }^{42,43}$ In patients with acute ischemia, CBF values greater than $20 \mathrm{ml} / 100 \mathrm{~g} / \mathrm{min}$ are associated with sustained tissue viability and reversible neurological deficit, whereas CBF values less than $10 \mathrm{ml} / 100 \mathrm{~g} / \mathrm{min}$ correlate with the eventual infarct volume. In addition, CBF values between 10 and $20 \mathrm{ml} / 100 \mathrm{~g} / \mathrm{min}$ have been associated with neurological deficits that have been reversible with the use of aggressive revascularization procedures, or with infarction if no revascularization occurred. ${ }^{13,14}$ Xenon-enhanced computed tomography scanning has been used to identify the presence of ischemia (defined by a CBF value $<20 \mathrm{ml} /$ $100 \mathrm{~g} / \mathrm{min}$ ) in patients with post-SAH vasospasm, and it has also been used to document improved CBF after medical or endovascular therapy. ${ }^{12,15,45}$

TABLE 1

Comparison of perfusion imaging techniques*

\begin{tabular}{|c|c|c|c|c|}
\hline Technique & $\begin{array}{l}\text { Perfusion } \\
\text { Parameters } \\
\text { Measured }\end{array}$ & $\begin{array}{l}\text { Other } \\
\text { Imaging } \\
\text { Obtained }\end{array}$ & Pros & Cons \\
\hline $\mathrm{Xe}-\mathrm{CT}$ & $\mathrm{CBF}$ & $\mathrm{CT}$ & $\begin{array}{l}\text { quantitative, standardized, } \\
\text { relatively inexpensive, } \\
\text { well-validated }\end{array}$ & $\begin{array}{l}\text { limited availability, medical-grade Xe } \\
\text { needs approval by FDA, requires } \\
\text { extreme patient cooperation }\end{array}$ \\
\hline SPECT & $\mathrm{CBF}$ & none & easy to perform, well-validated & $\begin{array}{l}\text { limited availability, semiquantitative, } \\
\text { low spatial resolution }\end{array}$ \\
\hline PCT & CBF, CBV, MTT & CT, CTA & $\begin{array}{l}\text { short scanning time, quantitative, } \\
\text { no special equipment needed }\end{array}$ & $\begin{array}{l}\text { not fully validated, limited } \\
\text { anatomical coverage, } \\
\text { radiation exposure, contrast load }\end{array}$ \\
\hline PWI/DWI & CBF, CBV, MTT & MRI, MRA & $\begin{array}{l}\text { images of entire brain, } \\
\text { assessment of tissue viability, } \\
\text { no radiation exposure }\end{array}$ & longer scanning time, semiquantitative \\
\hline
\end{tabular}

* $\mathrm{CTA}=\mathrm{CT}$ angiography; DWI = diffusion-weighted MR imaging; FDA = Food and Drug Administration; MRA = MR angiography; MTT = mean transit time; PCT = perfusion CT; PWI = perfusion-weighted MR imaging. 
Paired Xe-CT studies obtained before and after an acetazolamide vasodilatory stimulus have been used to assess hemodynamic impairment in patients with chronic ischemia. Decreased vascular response to the acetazolamide indicates preexisting vasodilation and loss of normal autoregulatory vascular reserve. A decrease in $\mathrm{CBF}$ values after acetazolamide administration indicates a high risk of subsequent stroke. ${ }^{28}$ In the setting of carotid balloon test occlusion, Xe-CT scanning has been used to help identify patients in whom the balloon test occlusion is clinically successful but who have a CBF value less than $30 \mathrm{ml} / 100$ $\mathrm{g} / \mathrm{min}$, placing them at increased risk for stroke. ${ }^{20}$ Adding acetazolamide may help identify patients who exhaust their cerebrovascular reserve during the balloon test occlusion.

Xenon-contrasted computed tomography is not only a quantitative method of CBF analysis but also is standardized for a given CT system and between different systems. Because it is an inert gas, xenon cannot cause allergic reactions, but at the concentration used for Xe-CT scanning, it produces mild sedation, sensory changes, and nausea. Patient motion can produce artifacts that hamper interpretation of the scans, and $\mathrm{CBF}$ is the only parameter that can be measured with the Xe-CT modality.

\section{The SPECT Modality}

In SPECT imaging, a radioisotope such as technetium$99 \mathrm{~m}$ is attached to a delivery compound that passes through the blood-brain barrier after intravenous injection and is metabolized by neuronal and glial cells. Common delivery compounds include hexamethylpropyleneamine oxime and ethyl cysteinate dimer. ${ }^{40}$ When the tracing agent is injected, the delivery compound's circulation to and localization within the cerebral tissues occur within 1 minute. This radioactive compound localizes during its first passage, with an uptake proportional to $\mathrm{CBF}$ at the moment of passage rather than the progressive tissue uptake that is characteristic of the xenon method described earlier. Therefore, SPECT imaging can be performed anytime within a few hours after injection of the delivery compound. Data are acquired in a $360^{\circ}$ rotation that requires approximately 5 minutes to complete. ${ }^{40}$

With this semiquantitative technique, activity values based on radioisotope signal levels in a given ischemic area are compared with similar activity in a presumably normal area in the contralateral cerebral hemisphere or with the presumably unaffected cerebellum, then the former values (ischemic) are divided by the latter (normal) to obtain the ratio (relative CBF). Of course, this formula assumes that the CBF to the unaffected hemisphere is normal, which frequently is not the case. This assumption obviously is incorrect for patients with chronic cerebrovascular disease, which is usually multifocal, or for patients with post-SAH vasospasm. Even in patients with acute stroke, complicated alterations of CBF in distant territories, even in the presumably unaffected hemisphere, can produce errors when such ratios are calculated..$^{18,44}$

The SPECT modality is a well-established technique with a sensitivity of 61 to $74 \%$ and a specificity to abnormal perfusion in acute stroke of 88 to $98 \% .^{23}$ It is a better modality for detecting cortical infarcts than smaller infarcts involving deep perforating vessels. The accuracy and reliability of SPECT in determining CBF have been shown by comparisons with other techniques, including PET and perfusion-weighted MR imaging, to a point at which there clearly is a linear relationship between relative and absolute CBF. ${ }^{21}$

The SPECT modality has been used in conjunction with an acetazolamide challenge to assess hemodynamic impairment in patients with chronic cerebrovascular disease. This approach requires the performance of two examinations separated by one or more days and assumes that CBF does not change significantly between the two tests. Relative (but not absolute) flow values can be obtained. Similarly, SPECT scanning has been used during carotid balloon test occlusion, with the radiotracer being injected while the balloon is inflated. Imaging can be obtained within several hours to document the relative $\mathrm{CBF}$ at the time of balloon occlusion. ${ }^{21}$

There have been limited reports of the use of the SPECT modality in patients with post-SAH vasospasm. ${ }^{7}$ Regional hypoperfusion on SPECT scans due to post-SAH vasospasm correlates with the presence and severity of DIND. This technology can provide early evidence of vasospasm-induced hypoperfusion and may help to differentiate this hypoperfusion from other causes of neurological deterioration.

The SPECT imaging modality is relatively accessible, with most large radiology departments having the appropriate hardware and software, but it may be difficult to make the radioactive injected compound on an emergency basis. It is a low-resolution technique compared with MR imaging and CT scanning, providing so little anatomical detail that it is necessary to correlate the findings with a higher-resolution imaging modality. Because SPECT is only a semiquantitative technique, it requires comparison with the contralateral "normal" hemisphere. Relative CBF is the only parameter that can be measured with this technique.

\section{The Dynamic Perfusion CT Modality}

Dynamic perfusion CT studies are obtained by monitoring the first pass of an iodinated contrast agent through the cerebral vasculature and measuring the changes in tissue attenuation. Postprocessing of the perfusion CT data allows the generation of color-coded maps of various perfusion parameters, including $\mathrm{CBF}, \mathrm{CBV}$, mean transit time, and the time to peak, which is the time from the start of contrast agent injection to the time of maximum enhancement. ${ }^{11}$ Perfusion CT is based on the central volume principle, which states that the CBF value is the ratio of the blood volume within all blood vessels in a given volume of tissue (CBV, which is measured in milliliters per gram) to the mean transit time (measured in seconds) of the contrast agent, from the arterial input to the venous drainage, within the volume being evaluated $(\mathrm{CBF}=\mathrm{CBV} /$ mean transit time). After intravenous contrast material injection, a time-density curve is constructed that indicates the change in density of the imaged tissues as the agent passes through the vasculature, and the area under that curve indicates the CBV. ${ }^{19}$ Calculation of mean transit time and $\mathrm{CBF}$ requires knowledge of the agent's concentration in the feeding arteries and draining veins related to the volume of tissue. Using mathematical models, the mean transit time and CBF can be approximated. ${ }^{3}$ 
With CT scans, the measurement of the change in tissue density following intravascular injection of a contrast agent can be used to calculate CBV.,16 The mean transit time and $\mathrm{CBF}$ are time-related values and require rapid imaging of a limited volume of imaged slices to determine the density change over time, arterial input, and venous outflow values, and to facilitate the use of mathematical models that assume that the contrast agent stays within the vasculature. The ability to measure these perfusion parameters with accuracy has been made possible with the development of high-speed helical and spiral CT scanners that have solid-state detectors and gantries designed to contain the very high gravitational forces generated by high-speed rotation of the x-ray tube. Software development has been critical to allowing the rapid electronic transfer of data from the detector arrays and rapid image reconstruction for perfusion analysis. ${ }^{3,16}$

In recent studies, researchers have indicated that perfusion CT scanning may be used to distinguish reversible from nonreversible ischemia by assessing quantitative perfusion parameter values or generating maps by using the quantitative data and special software. ${ }^{10}$ The latter method assumes that as $\mathrm{CBF}$ decreases, areas of reversible ischemia will have normal to increased CBV because of autoregulatory vasodilation, whereas areas of irreversible infarction will have decreased CBV. Thus, areas of low CBV may be comparable to areas of restricted diffusion on diffusion-weighted images obtained using MR imaging (Fig. 1). ${ }^{10,35}$ Initial studies attempting to define quantitative thresholds for areas of reversible ischemia and irreversible infarction produced values similar to those obtained with other perfusion imaging modalities such as PET and XeCT scanning.

Use of perfusion CT scanning in other clinical settings has been limited. It has been used in conjunction with acetazolamide challenge to assess cerebrovascular reserve. ${ }^{10,11}$ If perfusion pressure is not maintained, cerebral autoregulatory vasodilation occurs to maintain near-normal $\mathrm{CBF}$ with a resultant increase in $\mathrm{CBV} .{ }^{8}$ Paired CBF measurements before and after a vasodilatory stimulus such as acetazolamide can provide information about the presence of autoregulatory vasodilation and impaired reserve vasodilatory capacity. Acetazolamide normally causes vasodilation and at least a $25 \%$ increase in $\mathrm{CBF}^{46}$ However, vascular territories under hemodynamic stress are already maximally vasodilated and cannot respond further to acetazolamide. Values for CBF in these regions will not increase appropriately, and there may even be a decrease in $\mathrm{CBF}$ in the affected territory after acetazolamide injection, because vasodilation in other well-perfused brain territories diverts blood away from fully vasodilated, poorly perfused regions. Improvement in perfusion CT parameters after stent placement or bypass procedures has been demonstrated in case reports. ${ }^{10}$ Studies of the use of perfusion $\mathrm{CT}$ in patients with post-SAH vasospasm and those undergoing carotid balloon test occlusion are in preliminary stages. . $^{17,27}$

Perfusion CT is a rapid technique requiring approximately 1 minute of scanning time and several minutes to obtain postprocessed perfusion maps. It potentially can
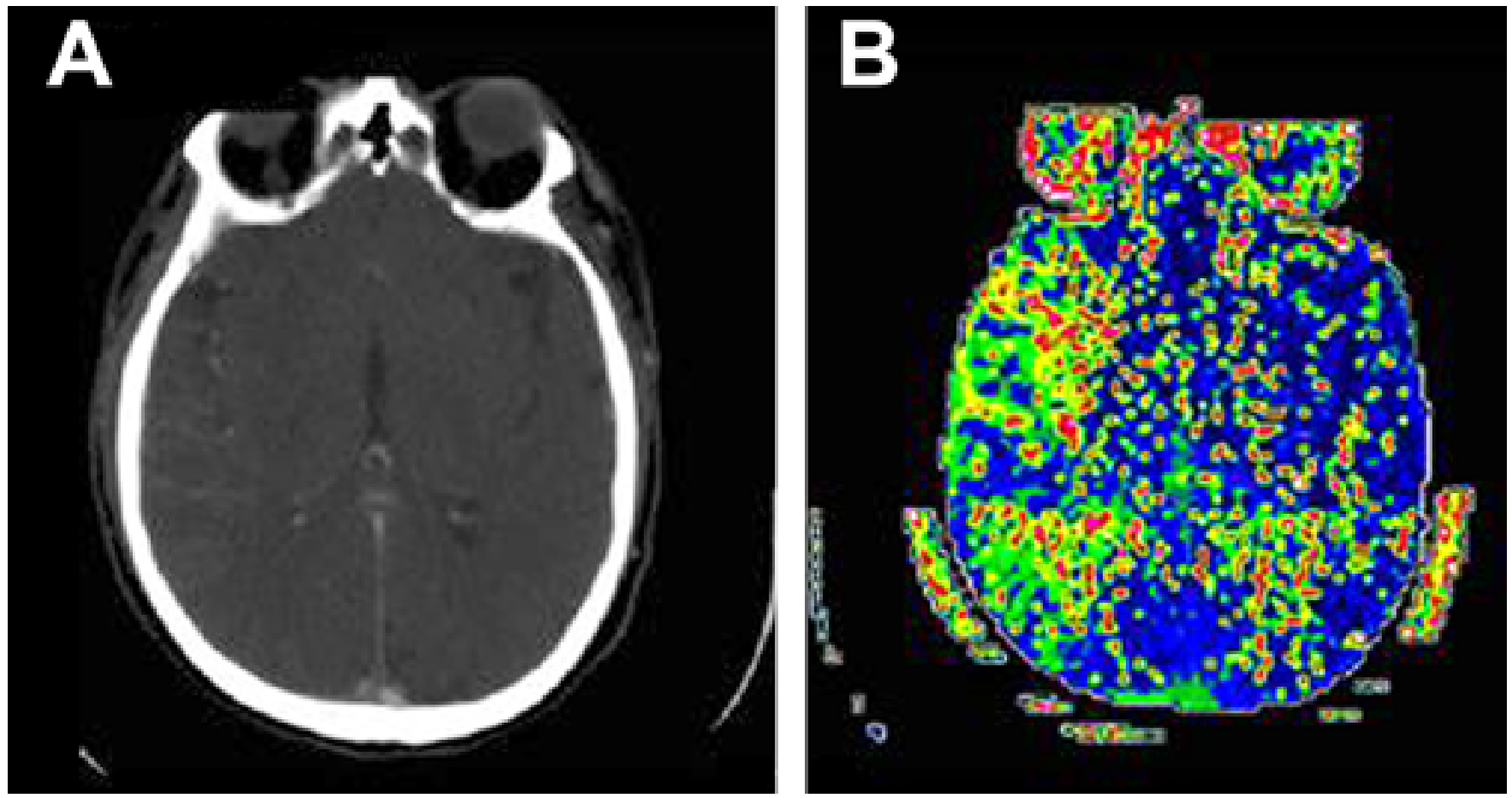

FIG. 1. Postoperative CT images obtained in a 52-year-old man 7 days after SAH and placement of a coil for a ruptured right posterior communicating artery aneurysm. A: Unenhanced CT head scan. B: Dynamic perfusion CT demonstrating decreased $\mathrm{CBF}$ in the right frontotemporal region consistent with right MCA hypoperfusion. Increased signal intensity in the right MCA territory indicates altered (decreased) blood flow in comparison with the contralateral hemisphere. 
be widely available because it requires only a helical CT scanner and appropriate software. Other CT examinations such as unenhanced CT scans and CT angiography studies can be obtained in the same setting; however, significant concerns exist regarding the accuracy and reproducibility of the quantitative values obtained with perfusion CT. For example, only a limited area of the brain can be imaged with this technique (typically a 10- to 30-mm-thick section, usually at the level of the basal ganglia), which limits visualization to portions of the ACA, MCA, and posterior cerebral artery territories. ${ }^{17}$ Perfusion CT scanning may be contraindicated in patients with allergies to contrast materials or compromised renal function. In recent papers, researchers have suggested that using the combination of CT angiography and CBV maps obtained using perfusion CT is a fast and effective way of evaluating ischemia and may provide opportunities to screen patients for early therapeutic vasospasm intervention following aneurysmal $\mathrm{SAH}$.

\section{The Perfusion- and Diffusion-Weighted MR Imaging Modalities}

Although perfusion- and diffusion-weighted MR imaging are distinctly different techniques, we will consider them together because they have interrelated physiological parameters and both usually are performed during the same imaging examination.

There are two types of perfusion MR imaging: dynamic susceptibility contrast imaging, which is analogous to perfusion CT, and the arterial spin labeling method. ${ }^{23} \mathrm{In}$ dynamic susceptibility contrast imaging, a paramagnetic contrast agent is used to produce dephasing of protons in an area equal to the radius of the blood vessel as the agent moves through the vasculature, which leads to an alteration of $\mathrm{T}_{2}$ relaxation in the adjoining tissues. The degree of $\mathrm{T}_{2}$ effect is proportional to the perfusion. ${ }^{34}$ In the arterial spin labeling method, water proton "spins" in the extracranial blood are saturated and inverted electromagnetically, and these labeled protons mix with extravascular water in the brain. Intracranial imaging before and after spin inversion is used to detect the difference in tissue magnetization, which is proportional to local perfusion. The arterial spin labeling method has the disadvantage of a relatively low signal-to-noise ratio, so it requires long imaging times as compensation and is still experimental. ${ }^{6}$

The most commonly used MR perfusion technique is dynamic susceptibility contrast imaging. It is similar to perfusion $\mathrm{CT}$ in that a contrast agent (a gadolinium chelate) that is injected intravenously causes changes in the MR signal as the agent passes through the vasculature. The change in signal intensity is then measured and perfusion maps are generated, most commonly of CBF, CBV, time to peak, or mean transit time.

Perfusion-weighted MR imaging is usually performed in conjunction with diffusion-weighted MR imaging and the assessment of apparent diffusion coefficient values. This combination of techniques can be used to differentiate ischemic from infarcted tissue and predict the death of ischemic tissue if reperfusion does not occur. ${ }^{21}$ Areas of abnormal diffusion usually are assumed to represent irreversible infarction. If an abnormality detected on perfusion-weighted MR imaging is larger than the area of restricted diffusion (diffusion/perfusion mismatch), then the region with normal diffusion but abnormal perfusion is called the ischemic penumbra. If flow is not restored to the penumbra, the diffusion abnormality presumably will increase in size to include much of the area of perfusion abnormality. ${ }^{21}$

More recent studies, however, have revealed a more complicated process. ${ }^{23,35}$ The diffusion changes may be partially reversible in patients undergoing successful treatment, such that the penumbra includes not only the area of diffusion/perfusion mismatch but also part of the area of restricted diffusion. ${ }^{23,35}$ Also, there is no consensus on which perfusion parameter or combination of parameters best defines the penumbra, although a decreased CBV likely indicates tissue that is destined to become infarcted. Perfusion-weighted MR imaging has had limited use in other clinical settings. There are some published reports describing its use in the assessment of patients with chronic cerebrovascular disease, including the use of acetazolamide challenge to evaluate cerebrovascular reserve. ${ }^{25}$

Both perfusion- and diffusion-weighted MR imaging are relatively time consuming, requiring 15 to 20 minutes of imaging time, even with limited stroke imaging protocols, and they are generally less available and more expensive than CT scanning. For most clinical uses, relative but not absolute quantitative perfusion maps (relative CBF, relative $\mathrm{CBV}$ ) can be obtained with MR imaging techniques. However, both perfusion- and diffusion-weighted MR imaging permit imaging of the entire brain, provide information on perfusion, and can be used to assess vascular patency if MR angiography is added. Also, diffusion-weighted MR imaging can provide information on the status of tissue viability. No ionizing radiation is used, and there are usually fewer contraindications to gadolinium contrast agents than to iodinated contrast. ${ }^{23}$

Recently, the use of perfusion-weighted MR imaging in evaluating patients with post-SAH vasospasm was examined. ${ }^{33}$ Results in patients with vasospasm show that diffusion-weighted MR imaging can be used to detect small regions of early ischemic injury within large regions of abnormal relative $\mathrm{CBF}$ and mean transit time. Widespread decreases in relative CBF and mean transit time occurred in each patient throughout regions supplied by vessels with angiographically demonstrated vasospasm. In contrast, the relative CBV maps were normal except in a patient in whom a large infarct had already occurred. These MR data were concordant with PET data that demonstrated a mismatch between blood flow and blood volume in regions affected by post-SAH vasospasm (Fig. 2). One interpretation of this pattern in patients with vasospasm and in those with acute ischemic stroke is that increased collateral flow through maximally dilated microcirculation preserves $\mathrm{CBV}$ in regions with reduced $\mathrm{CBF} .{ }^{37}$

In patients with acute stroke, this pattern of normal relative $\mathrm{CBV}$, decreased relative $\mathrm{CBF}$, and increased mean transit time occurred around large regions of decreased relative CBV that produced abnormal diffusion-weighted MR images ${ }^{37}$ In contrast, in patients with post-SAH vasospasm, a less severe decrease in relative CBF with preserved relative $\mathrm{CBV}$ was found to be the predominant abnormality ${ }^{33}$ In one study, large areas of brain with decreased relative $\mathrm{CBF}$ and increased mean transit time were seen on perfusion-weighted MR imaging in all patients with clinically symptomatic vasospasm, and the re- 
S. P. Lad, et al.

\section{Diffusion}

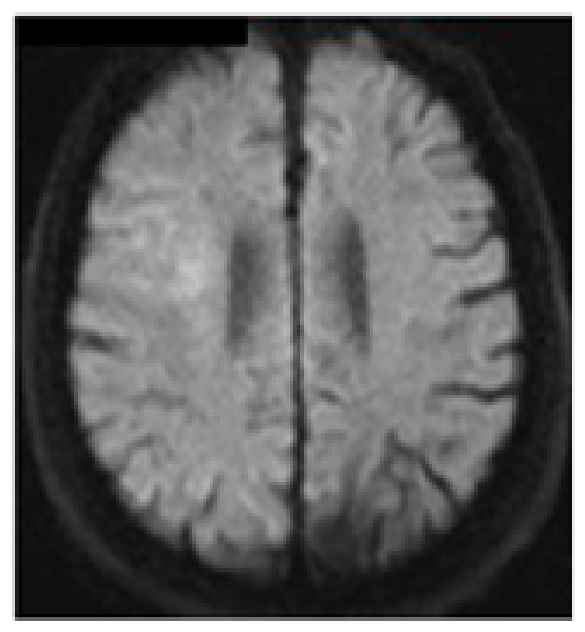

\section{SPECT}

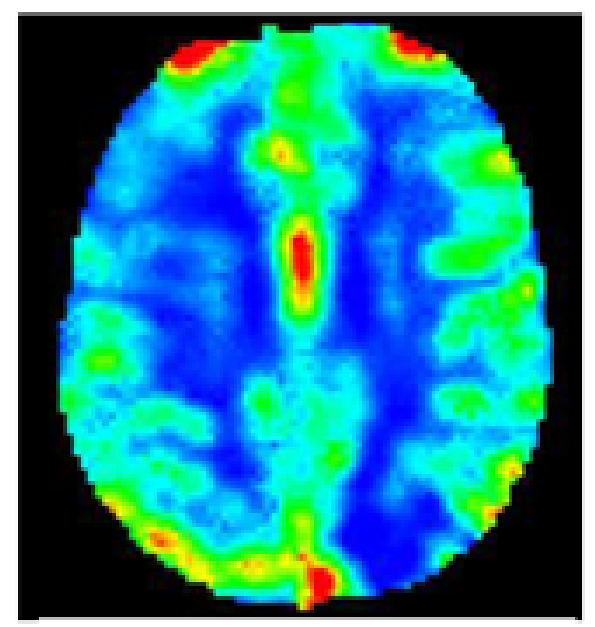

\section{Perfusion}

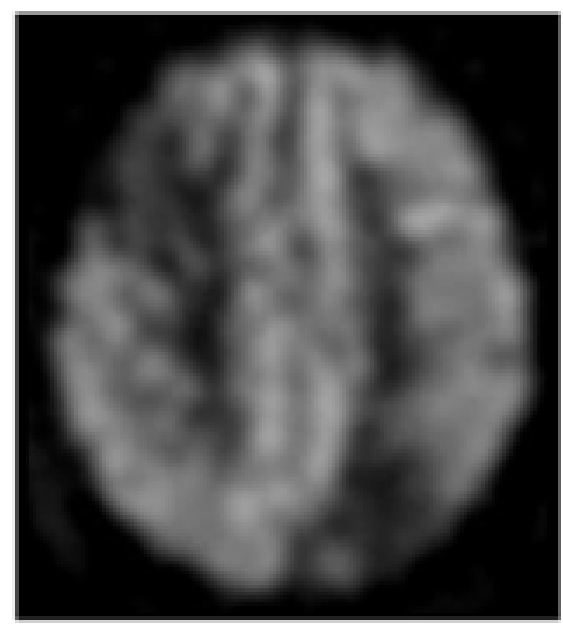

\section{PET}

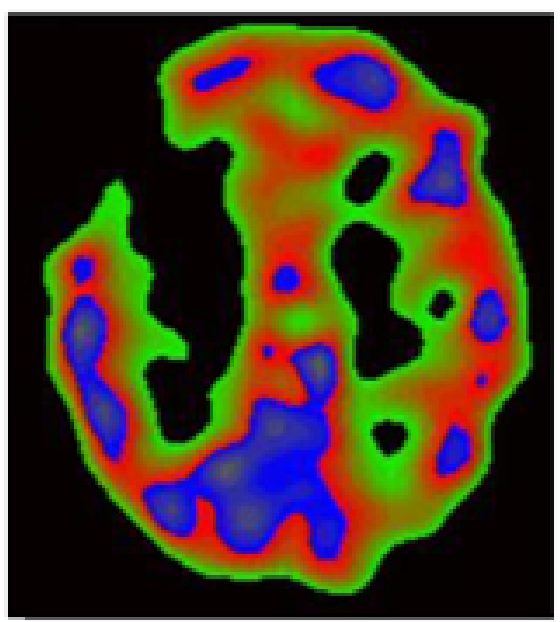

FIG. 2. Comparison of diffusion- and perfusion-weighted MR imaging, SPECT scans, and PET studies in a 45-yearold man 10 days after clip placement for an aneurysm rupture of the right $\mathrm{M}_{2}$ segment of the MCA. Upper Left: Axial diffusion-weighted MR image demonstrating very subtle changes in the right frontal and periventricular white matter consistent with ischemia. Upper Right: Axial perfusion-weighted MR image demonstrating a map of mean transit time with decreased flow to the right frontal lobe encompassing a portion of the right MCA territory. This is consistent with a diffusion/perfusion mismatch. Lower Left: Axial SPECT image demonstrating decreased levels of CBF in the right frontal region in comparison with a similar area on the left. Lower Right: Axial PET image confirming decreased uptake in the right frontal lobe consistent with evolving ischemic injury.

gional location of these abnormalities correlated well with angiographic findings. The patients were receiving triple$\mathrm{H}$ therapy during the scans for their suspected symptomatic vasospasm, and the effect of the treatment on the hemodynamic pattern is unknown. In the patients who were treated aggressively to prevent infarction, the large areas of abnormal relative $\mathrm{CBF}$ and mean transit time did not evolve into infarction and were normal on the followup studies. In most patients, the neurological signs and symptoms correlated better with the anatomy of the blood flow abnormality than with the small regions of infarction. The flow reductions also were probably longstanding, given that in some patients there was a long duration between symptom onset and scan. This finding underlies the sensitivity of perfusion-weighted MR imaging to detect levels of ischemia that might cause neurological deficits but that are not severe enough to produce an infarct (Fig. 3). ${ }^{4}$

\section{Conclusions}

The development of cerebral perfusion imaging technologies during the last several decades has been exciting; however, their role in the evaluation of a variety of diseases of the central nervous system is still controversial. Several disorders of blood flow, such as acute stroke, chronic vascular occlusive disease, and post-SAH vaso- 

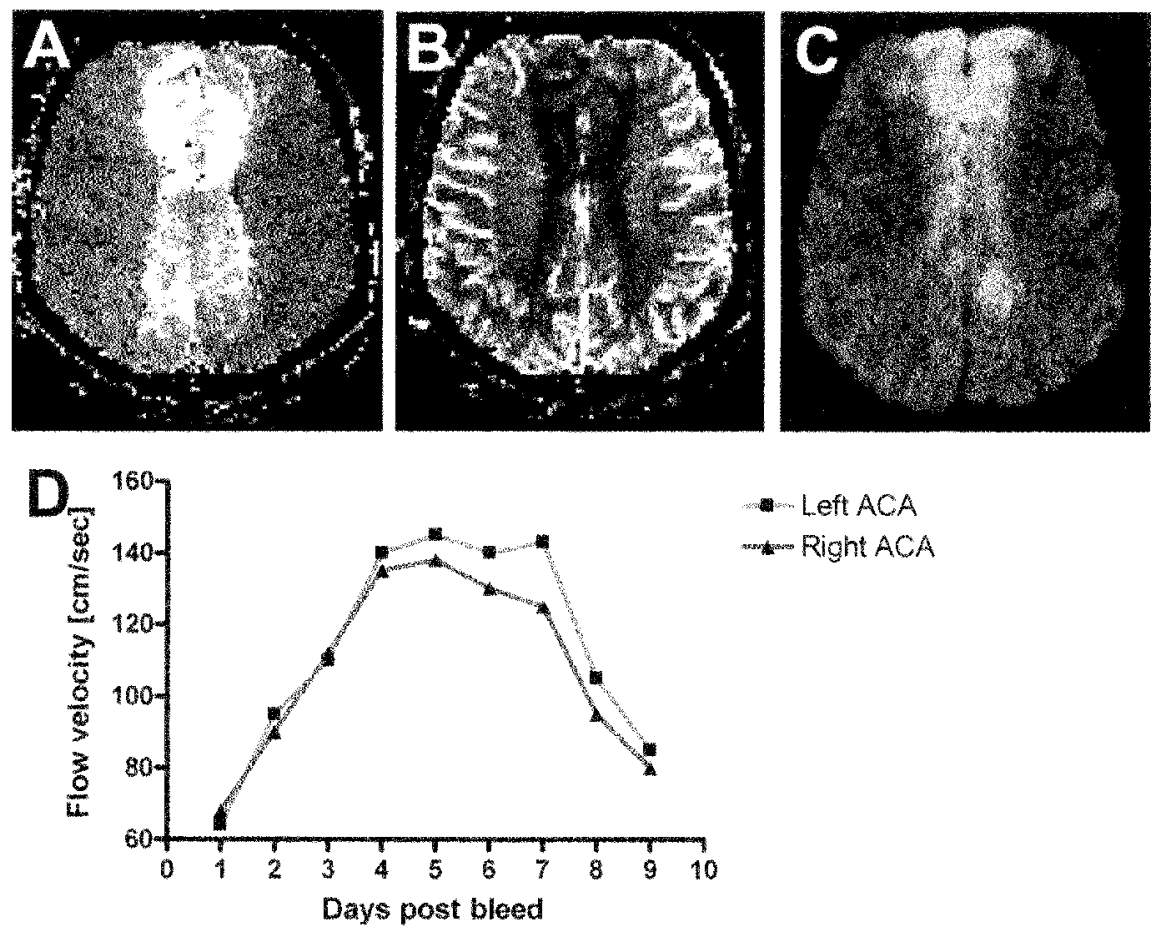

Left ACA

Right ACA
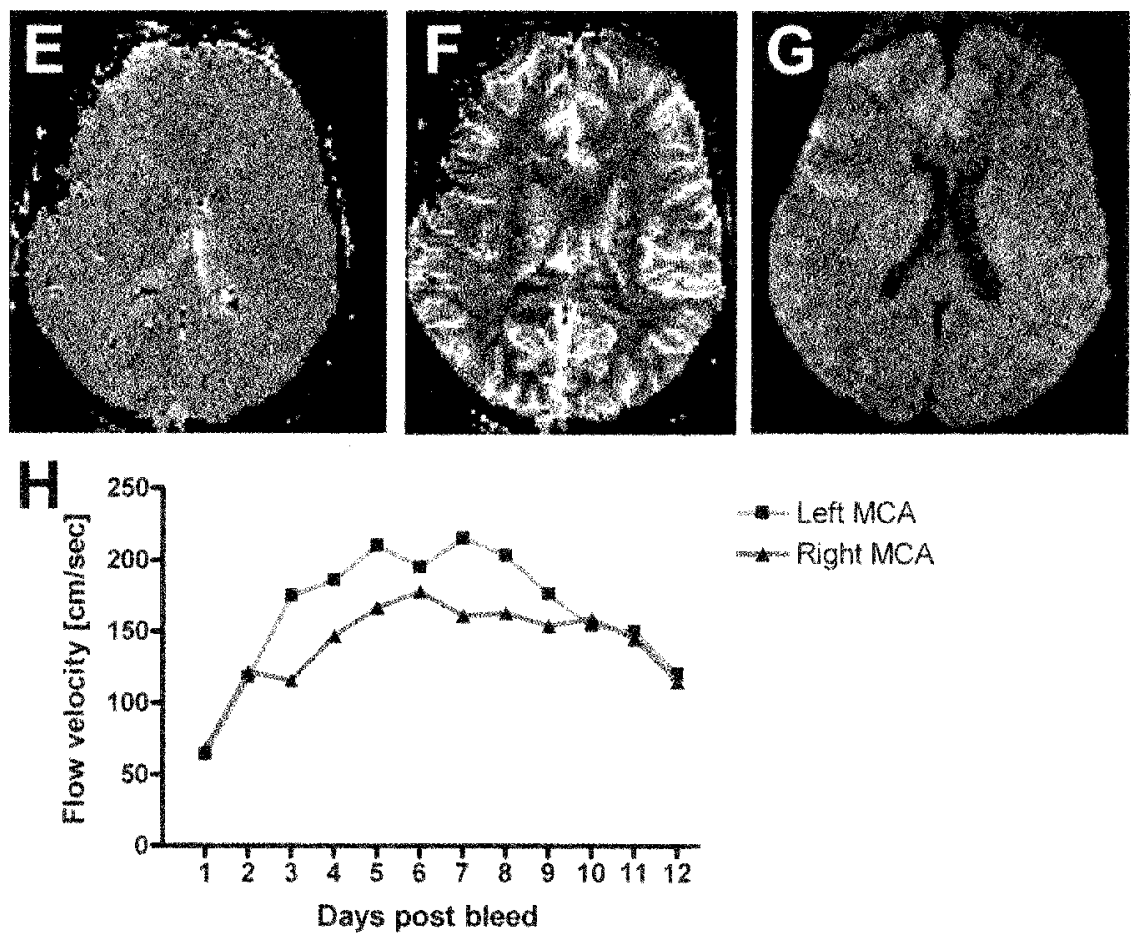

FIG. 3. A-D: Perfusion images and graphs relating to a 55-year-old man who underwent coil occlusion of a ruptured anterior communicating artery aneurysm. The patient developed increased left and right ACA velocities, as demonstrated on TCD ultrasonography. A and B: Perfusion-weighted MR images demonstrating bilateral hypoperfusion in the ACA distributions. C: Diffusion-weighted MR image obtained 2 days later demonstrating bilateral ACA infarctions. D: Graph of ACA velocities compiled using data obtained on TCD ultrasonography. Velocities have increased to greater than $130 \mathrm{~cm} / \mathrm{second}$ at 4 days post-SAH. In this case, perfusion-weighted MR imaging helped determine that this patient would develop DIND. E-H: Perfusion images and graph relating to a 40-year-old man with SAH who underwent coil placement in a left MCA aneurysm. He developed significantly increased TCD velocities and underwent perfusionweighted MR imaging. E and F: Perfusion-weighted MR images revealing no hypoperfusion 7 days after hemorrhage. G: Diffusion-weighted MR image reveals no evidence of brain infarction 9 days post-SAH. H: Graph of MCA velocities compiled using data obtained on TCD ultrasonography. Velocities have increased to greater than $200 \mathrm{~cm} / \mathrm{second}$. However, the brain was able to compensate and DINDs did not develop. 
spasm are beginning to be analyzed using various perfusion imaging techniques. The accuracy, reproducibility, and reliability of the data obtained using these techniques have been evaluated in animal models and patients, and in general have been found to be of very high quality. The use of cerebral perfusion imaging techniques for the early detection of vasospasm is emerging and may provide opportunities for early therapeutic intervention to counteract vasospasm in its earliest stages and prevent the occurrence of DINDs.

\section{References}

1. Aaslid R, Huber P, Nornes H: Evaluation of cerebrovascular spasm with transcranial Doppler ultrasound. J Neurosurg 60: 37-41, 1984

2. Aaslid R, Markwalder TM, Nornes H: Noninvasive transcranial Doppler ultrasound recording of flow velocity in basal cerebral arteries. J Neurosurg 57:769-774, 1982

3. Aksoy FG, Lev MH: Dynamic contrast-enhanced brain perfusion imaging: technique and clinical applications. Semin Ultrasound CT MR 21:462-477, 2000

4. Ay H, Buonanno FS, Rordorf G, Schaefer PW, Schwamm LH, $\mathrm{Wu} \mathrm{O}$, et al: Normal diffusion-weighted MRI during stroke-like deficits. Neurology 52:1784-1792, 1999

5. Bell TE, LaGrange KM, Maier CM, Steinberg GK: Transcranial Doppler: correlation of blood velocity measurement with clinical status in subarachnoid hemorrhage. J Neurosci Nurs 24:215-219, 1992

6. Chalela JA, Alsop DC, Gonzalez-Atavales JB, Maldjian JA, Kasner SE, Detre JA: Magnetic resonance perfusion imaging in acute ischemic stroke using continuous arterial spin labeling. Stroke 31:680-687, 2000

7. Davis SM, Andrews JT, Lichtenstein M, Rossiter SC, Kaye AH, Hopper J: Correlations between cerebral arterial velocities, blood flow, and delayed ischemia after subarachnoid hemorrhage. Stroke 23:492-497, 1992

8. Derdeyn CP, Grubb RL Jr, Powers WJ: Cerebral hemodynamic impairment: methods of measurement and association with stroke risk. Neurology 53:251-259, 1999

9. DeWitt DS, Fatouros PP, Wist AO, Stewart LM, Kontos HA, Hall JA, et al: Stable xenon versus radiolabeled microsphere cerebral blood flow measurements in baboons. Stroke 20: 1716-1723, 1989

10. Eastwood JD, Alexander MJ, Petrella JR, Provenzale JM: Dynamic CT perfusion imaging with acetazolamide challenge for the preprocedural evaluation of a patient with symptomatic middle cerebral artery occlusive disease. AJNR Am J Neuroradiol 23:285-287, 2002

11. Eastwood JD, Lev MH, Provenzale JM: Perfusion CT with iodinated contrast material. AJR Am J Roentgenol 180:3-12, 2003

12. Firlik AD, Kaufmann AM, Jungreis CA, Yonas H: Effect of transluminal angioplasty on cerebral blood flow in the management of symptomatic vasospasm following aneurysmal subarachnoid hemorrhage. J Neurosurg 86:830-839, 1997

13. Firlik AD, Kaufmann AM, Wechsler LR, Firlik KS, Fukui MB, Yonas H: Quantitative cerebral blood flow determinations in acute ischemic stroke. Relationship to computed tomography and angiography. Stroke 28:2208-2213, 1997

14. Firlik AD, Rubin G, Yonas H, Wechsler LR: Relation between cerebral blood flow and neurologic deficit resolution in acute ischemic stroke. Neurology 51:177-182, 1998

15. Firlik KS, Kaufmann AM, Firlik AD, Jungreis CA, Yonas $\mathrm{H}$ : Intra-arterial papaverine for the treatment of cerebral vasospasm following aneurysmal subarachnoid hemorrhage. Surg Neurol 51:66-74, 1999
16. Hamberg LM, Hunter GJ, Halpern EF, Hoop B, Gazelle GS, Wolf GL: Quantitative high-resolution measurement of cerebrovascular physiology with slip-ring CT. AJNR Am J Neuroradiol 17:639-650, 1996

17. Hoeffner EG, Case I, Jain R, Gujar SK, Shah GV, Deveikis JP, et al: Cerebral perfusion CT: technique and clinical applications. Radiology 231:632-644, 2004

18. Hughes RL, Yonas H, Gur D, Latchaw R: Cerebral blood flow determination within the first 8 hours of cerebral infarction using stable xenon-enhanced computed tomography. Stroke 20:754-760, 1989

19. Hunter GJ, Hamberg LM, Ponzo JA, Huang-Hellinger FR, Morris PP, Rabinov J, et al: Assessment of cerebral perfusion and arterial anatomy in hyperacute stroke with three-dimensional functional CT: early clinical results. AJNR Am J Neuroradiol 19:29-37, 1998

20. Johnson DW, Stringer WA, Marks MP, Yonas H, Good WF, Gur D: Stable xenon CT cerebral blood flow imaging: rationale for and role in clinical decision making. AJNR Am J Neuroradiol 12:201-213, 1991

21. Karonen JO, Liu Y, Vanninen RL, Ostergaard L, Kaarina Partanen PL, Vainio PA, et al: Combined perfusion- and diffusionweighted MR imaging in acute ischemic stroke during the 1st week: a longitudinal study. Radiology 217:886-894, 2000

22. Lassen NA, Ingvar DH: Radioisotopic assessment of regional cerebral blood flow. Prog Nucl Med 1:376-409, 1972

23. Latchaw RE: Cerebral perfusion imaging in acute stroke. $\mathbf{J}$ Vasc Interv Radiol 15: S29-S46, 2004

24. Lee KH, Lukovits T, Friedman JA: "Triple-H" therapy for cerebral vasospasm following subarachnoid hemorrhage. Neurocrit Care 4:68-76, 2006

25. Lythgoe DJ, Ostergaard L, William SC, Cluckie A, BuxtonThomas M, Simmons A, et al: Quantitative perfusion imaging in carotid artery stenosis using dynamic susceptibility contrastenhanced magnetic resonance imaging. Magn Reson Imaging 18: $1-11,2000$

26. Marks MP, Steinberg GK, Lane B: Intraarterial papaverine for the treatment of vasospasm. AJNR Am J Neuroradiol 14: 822-826, 1993

27. Nabavi DG, LeBlanc LM, Baxter B, Lee DH, Fox AJ, Lownie $\mathrm{SP}$, et al: Monitoring cerebral perfusion after subarachnoid hemorrhage using CT. Neuroradiology 43:7-16, 2001

28. Nariai T, Suzuki R, Hirakawa K, Maehara T, Ishii K, Senda M: Vascular reserve in chronic cerebral ischemia measured by the acetazolamide challenge test: comparison with positron emission tomography. AJNR Am J Neuroradiol 16:563-570, 1995

29. Naval NS, Stevens RD, Mirski MA, Bhardwaj A: Controversies in the management of aneurysmal subarachnoid hemorrhage. Crit Care Med 34:511-524, 2006

30. Pindzola RR, Yonas H: The xenon-enhanced computed tomography cerebral blood flow method. Neurosurgery 43: 1488-1492, 1998

31. Provencio JJ, Vora N: Subarachnoid hemorrhage and inflammation: bench to bedside and back. Semin Neurol 25:435-444, 2005

32. Rabinstein AA, Friedman JA, Weigand SD, McClelland RL, Fulgham JR, Manno EM, et al: Predictors of cerebral infarction in aneurysmal subarachnoid hemorrhage. Stroke 35: 1862-1866, 2004

33. Rordorf G, Koroshetz WJ, Copen WA, Gonzalez G, Yamada K, Schaefer PW, et al: Diffusion- and perfusion-weighted imaging in vasospasm after subarachnoid hemorrhage. Stroke 30: 599-605, 1999

34. Rother J, Guckel F, Neff W, Schwartz A, Hennerici M: Assessment of regional cerebral blood volume in acute human stroke by use of single-slice dynamic susceptibility contrast-enhanced magnetic resonance imaging. Stroke 27:1088-1093, 1996

35. Schaefer PW, Hassankhani A, Putman C, Sorensen AG, 


\section{Cerebral perfusion imaging in vasospasm}

Schwamm L, Koroshetz W, et al: Characterization and evolution of diffusion MR imaging abnormalities in stroke patients undergoing intra-arterial thrombolysis. AJNR Am J Neuroradiol 25:951-957, 2004

36. Sehba FA, Bederson JB: Mechanisms of acute brain injury after subarachnoid hemorrhage. Neurol Res 28:381-398, 2006

37. Sorensen AG, Copen WA, Ostergaard L, Buonanno FS, Gonzalez RG, Rordorf G, et al: Hyperacute stroke: simultaneous measurement of relative cerebral blood volume, relative cerebral blood flow, and mean tissue transit time. Radiology 210: 519-527, 1999

38. Steinberg GK, Vanefsky MA, Marks MP, Adler JR Jr, Koenig $\mathrm{GH}$ : Failure of intracisternal tissue plasminogen activator to prevent vasospasm in certain patients with aneurysmal subarachnoid hemorrhage. Neurosurgery 34:809-814, 1994

39. Tani E: Molecular mechanisms involved in development of cerebral vasospasm. Neurosurg Focus 12(3): E1, 2002

40. Walovitch RC, Cheesman EH, Maheu LJ, Hall KM: Studies of the retention mechanism of the brain perfusion imaging agent 99mTc-bicisate (99mTc-ECD). J Cereb Blood Flow Metab 14 Suppl 1:S4-S11, 1994

41. White H, Venkatesh B: Applications of transcranial Doppler in the ICU: a review. Intensive Care Med 32:981-984, 2006

42. Yonas H, Darby JM, Marks EC, Durham SR, Maxwell C: CBF measured by Xe-CT: approach to analysis and normal values. J Cereb Blood Flow Metab 11:716-725, 1991

43. Yonas H, Good WF, Gur D, Wolfson SK Jr, Latchaw RE, Good $\mathrm{BC}$, et al: Mapping cerebral blood flow by xenon-enhanced computed tomography: clinical experience. Radiology 152: 435-442, 1984

44. Yonas H, Gur D, Claassen D, Wolfson SK Jr, Moossy J: Stable xenon enhanced computed tomography in the study of clinical and pathologic correlates of focal ischemia in baboons. Stroke 19:228-238, 1988

45. Yonas H, Sekhar L, Johnson DW, Gur D: Determination of irreversible ischemia by xenon-enhanced computed tomographic monitoring of cerebral blood flow in patients with symptomatic vasospasm. Neurosurgery 24:368-372, 1989

46. Yonas H, Smith HA, Durham SR, Pentheny SL, Johnson DW: Increased stroke risk predicted by compromised cerebral blood flow reactivity. J Neurosurg 79:483-489, 1993

Manuscript received June 16, 2006.

Accepted in final form August 4, 2006.

Address reprint requests to: Gary K. Steinberg, M.D., Ph.D., Department of Neurosurgery, Stanford University Medical Center, Edwards Building, Room R-281, 300 Pasteur Drive, Stanford, California 94305. email: gsteinberg@stanford.edu. 